\title{
Other and Unspecified Major Salivary Glands ICD-O-3
}

National Cancer Institute

\section{Source}

National Cancer Institute. Other and Unspecified Major Salivary Glands ICD-O-3. NCI

Thesaurus. Code C148340.

Major salivary glands that are referenced in topog raphic category C08 of ICD-O-3. 